\title{
The existence of almost periodic solution: via coincidence degree theory
}

\section{Sanfu Wang ${ }^{*}$}

\section{"Correspondence:}

sanfuwang@126.com

School of Electronic and

Information Engineering, Xi'an

Jiaotong University, Xian, 710000 ,

China

School of Mathematics and

Statistics, Tianshui Normal

University, Tianshui, 741001, China

\begin{abstract}
In the present paper, a new method is developed to study the existence of an almost periodic solution for the ordinary or functional differential equations. The approaches are based on topological degree and novel estimation techniques for the a priori bounds of unknown solutions for $L x=\lambda N x$. To investigate the existence of an almost periodic solution, a few good methods have been presented in the previous literature (such as using the Lyapunov function, averaging, exponential dichotomy, stability, separate conditions, and so on). But topological degree theory was never employed to study the almost periodic differential equations. Though Mawhin's coincidence degree is employed to study the existence of periodic differential equations extensively, it cannot be applied to study the almost periodic systems immediately. Some essentially new and interesting lemmas should be proved before applying topological degree theory to almost periodic systems. To the best knowledge of the authors', it is the first time that topological degree theory is employed to study the existence of almost periodic solution and this method can be seen as a good supplement to the known methods. Therefore, it will be of great significance to study the almost periodic systems by using this method. The approach followed in the paper could be further generalized to investigate the existence of almost periodic oscillatory in some other nonlinear dynamical systems. It is believed that it can be applied to image patterns, digital image processing, data processing, signal sparse decomposition and information technology, etc.
\end{abstract}

MSC: 34B15; 34C27; 34K14

Keywords: almost periodic solution; topological degree

\section{Introduction}

The existence of almost periodic solutions of ordinary differential equations has been discussed extensively in theory and in practice (for example, see [1-48] and the references cited therein). In particularly, many useful methods are developed to study the almost periodic differential equations in the classical references such as Hale [1-4], Fink [5-7], Yoshizawa [8, 9], Hino et al. [10], Seifert [11-14], Copple [15], Kato [16], Sell [17, 18], He [19], Favard [20], Bohr and Neugebauer [21], and Lakshmikantham and Leela [22]. We summarize their methods to study the existence of an almost periodic solution for the differential equations as six big categories:

(I) By using the semi-separated condition or the separated condition (considering the hull system), including the famous theorem (each hull system has a unique solution in $S$,

(c) 2016 Wang. This article is distributed under the terms of the Creative Commons Attribution 4.0 International License (http://creativecommons.org/licenses/by/4.0/), which permits unrestricted use, distribution, and reproduction in any medium, provided you give appropriate credit to the original author(s) and the source, provide a link to the Creative Commons license, and indicate if changes were made. 
then these solutions are all almost periodic, see He [19], Theorem 32, and Fink [5], Theorem 10.1).

(II) By using the Lyapunov function.

(III) By using the stability, including (weakly) quasi-uniformly asymptotic stability, total stability, stability under the perturbation of the hull (see Fink [5], Yoshizawa [8], and Kato [16]), relatively weakly uniformly asymptotic stability, and relatively total stability (see Hino et al. [10]).

(IV) By using the relations between asymptotically almost periodic function and the almost periodic functions.

(V) By combining the exponential dichotomy and fixed point theory including the Banach fixed point, Schauder fixed point, Lerry-Schauder fixed point approaches, and so on.

(VI) By the average method.

(VII) By using the smallest solution with respect to the norm (see Favard [20]).

(VIII) By the comparison method (see Fink [5] and Lakshmikantham and Leela [22]).

These methods have great significance in the study of the almost periodic differential equations and thus have many application in the specific systems arising from biology, neural networks, physics, chemistry, engineering, and so on (one can refer to [23-48]).

Though so many good methods were developed and applied to study the almost periodic equations, there is no paper studying the existence of almost periodic solutions by using topological degree theory. It is well known that topological degree theory is a powerful tool to study the periodic differential equations. It is usually used to study the existence of periodic solutions for the boundary value problem, the initial value problem, the twopoint boundary value problem and so on (for examples, one can refer to $[41,47,48]$ and references cited therein). Since topological degree theory plays such a great role in the periodic differential equations, it is natural to ask the question:

Can topological degree theory be employed to study the almost periodic differential equations? If so, how should one use topological degree theory to study the almost periodic systems?

Therefore, the present paper is devoted to giving an affirmative reply to the question. Topological degree theory can be applied to study the almost periodic nonlinear systems including the ordinary differential equations and functional differential equations. However, the method used to study the periodic systems cannot be applied to an almost periodic system directly. The method used in previous work (e.g. [41]) cannot be applied to the almost periodic system directly. Therefore, many definitions should be modified and many indispensable and essentially new lemmas should be proved to suit for the almost periodic systems.

\section{Preliminary}

In this section, in order to obtain the existence of almost periodic solutions of the differential equations, we shall make some preparation. For convenience, we first summarize in the following a few concepts and prove some preliminary results on almost periodic functions and topological degree theory that will be basic for the next section.

Definition 2.1 $x(t): R \rightarrow R^{n}$, which is continuous in $t$ and $x(t)$ is said to almost periodic on $R$, if, for any $\varepsilon>0$, it is possible to find a real number $l(\varepsilon)>0$ such that in any interval 
of length $l(\varepsilon)$ there exists $\tau=\tau(\varepsilon)$ such that the inequality

$$
\|f(t+\tau)-f(t)\|<\varepsilon
$$

is satisfied for all $t \in(-\infty,+\infty)$. The number $\tau$ is called a $\varepsilon$-translation number of $f(t)$ and $l(\varepsilon)$ is called the length of inclusion interval $T(f, \varepsilon)$.

Definition 2.2 A function $f(t, x)=\left(f_{1}(t, x), f_{2}(t, x), \ldots, f_{n}(t, x)\right)^{T}$, where $f(t, x)$ is an $n$-vector, $t$ is a real variable, and $x=\left(x_{1}(t), x_{2}(t), \ldots, x_{n}(t)\right)^{T}$ is an $n$-vector, is said to be almost periodic in $t$ uniformly with respect to $x \in S \subset R^{n}$, if $f(t, x)$ is continuous in $t \in R$ and $x \in S$, and if, for any $\varepsilon>0$, it is possible to find a constant $l(\varepsilon, S)>0$ such that in any interval of length $l(\varepsilon, S)$ there exists $\tau$ such that the inequality

$$
\|f(t+\tau, x)-f(t, x)\|=\sum_{i=1}^{n}\left|f_{i}(t+\tau, x)-f_{i}(t, x)\right|<\varepsilon
$$

is satisfied for all $t \in(-\infty,+\infty), x \in S$. The number $\tau$ is called a $\varepsilon$-translation number of $f(t, x)$ and $l(\varepsilon, S)$ is called the length of inclusion interval of $T(f, \varepsilon, S)$.

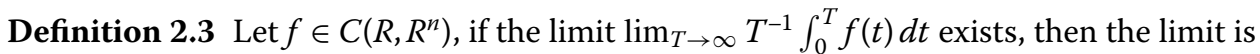
called the mean value of $f$. Denote $m(f(t))=\lim _{T \rightarrow \infty} T^{-1} \int_{0}^{T} f(t) d t$.

Obviously, $m(a)=a$ and $m(m(f(t)))=m(f(t))$, where $a \in R^{n}$ is a constant vector.

Lemma $2.1[5,8,19]$

(a) Let $f(t) \in C\left(R, R^{n}\right), f(t)$ is almost periodic if only if $x(t)$ is bounded and uniformly continuous on $R$.

(b) Let $f: R \times S \rightarrow R^{n}$ be almost periodic in $t$ uniformly with respect to $x \in S \subset R^{n}$, where $S$ is any compact set of $R^{n}$. Then $f(t, x)$ is bounded on $R \times S$ and uniformly continuous.

(c) Let $f(t)$ and $g(t)$ be all almost periodic, then $f(t)+g(t)$ is almost periodic. Moreover, if $\inf _{t \in R}|g(t)|>0$, then $f(t) / g(t)$ is also almost periodic.

Lemma $2.2[5,8,19]$ Suppose that $x \in C\left(R, R^{n}\right)$ is an almost periodic function. Then

$$
\Phi(t)=\int_{0}^{t} x(s) d s
$$

is almost periodic, if only if $\Phi(t)$ is bounded.

Lemma 2.3 $[5,8,19]$ (mean value theorem) Suppose that $f \in C\left(R, R^{n}\right)$ is an almost periodic function, then $m(f(t))$ exists, i.e. $\|m(f(t))\|<+\infty$. Moreover, if $f(t) \geq 0$ and $f(t) \not \equiv 0$, then $m(f(t))>0$.

Lemma 2.4 [47] (continuation theorem) Let $\Omega \subset X$ be an open and bounded set. Let $L$ be a Fredholm mapping of index zero and $N$ be L-compact on $\bar{\Omega}$. Assume

(a) for each $\lambda \in(0,1), x \in \partial \Omega \cap \operatorname{Dom} L, L x \neq \lambda N x$;

(b) for each $x \in \partial \Omega \cap \operatorname{Ker} L, Q N x \neq 0$; 
(c) $\operatorname{deg}\{J Q N, \Omega \cap \operatorname{Ker} L, 0\} \neq 0$.

Then $L x=N x$ has at least one solution in $\bar{\Omega} \cap \operatorname{Dom} L$.

In the following we shall introduce some function spaces and their norms, which are valid throughout this paper. Denote

$$
\begin{aligned}
& A P\left(R, R^{n}\right)=\left\{x(t)=\left(x_{1}(t), x_{2}(t), \ldots, x_{n}(t)\right)^{T} \mid x(t) \in C\left(R, R^{n}\right), x(t) \text { is almost periodic }\right\}, \\
& X=\left\{x(t) \in C\left(R, R^{n}\right) \mid x(t) \in A P\left(R, R^{n}\right), \dot{x}(t) \in A P\left(R, R^{n}\right)\right\}, \\
& Z=\left\{x(t) \in C\left(R, R^{n}\right) \mid x(t) \in A P\left(R, R^{n}\right)\right\} .
\end{aligned}
$$

The norms are given by

$$
\begin{aligned}
& \left|x_{i}(t)\right|_{0}=\sup _{t \in R}\left|x_{i}(t)\right|, \quad\left|x_{i}(t)\right|_{1}=\left|x_{i}(t)\right|_{0}+\left|\dot{x}_{i}(t)\right|_{0}, \quad i=1,2, \ldots, n, \\
& \|x(t)\|_{0}=\max _{1 \leq i \leq n}\left\{\left|x_{i}(t)\right|_{0}\right\}, \quad\|x(t)\|_{1}=\|x(t)\|_{0}+\|\dot{x}(t)\|_{0}=\max _{1 \leq i \leq n}\left\{\left|x_{i}(t)\right|_{1}\right\} .
\end{aligned}
$$

Obviously, $X$ and $Z$, respectively, endowed with the norms $\|\cdot\|_{1}$ and $\|\cdot\|_{0}$ are Banach spaces.

To proceed our study of the existence of almost periodic solutions, we need to prove the following important lemmas.

Lemma 2.5 If $x(t) \in A P\left(R, R^{n}\right)$, then there exists $t_{0} \in R$ such that $x\left(t_{0}\right)=\sup _{t \in R} x(t)$.

Proof In view of Lemma 2.1(a), $x(t)$ is bounded. Let $M=\sup _{t \in R} x(t)$. By way of contradiction, suppose that $x(t)<M$ for all $t \in R$. Obviously, $M-x(t)>0$ is also a continuously almost periodic function on $R$. Note that $\inf _{t \in R}(M-x(t))>0$. Hence, it follows from Lemma 2.1(c) that

$$
\frac{1}{M-x(t)} \text { is also an almost periodic function. }
$$

By Lemma 2.1(a) again, $\frac{1}{M-x(t)}$ is bounded. That is, there exists a constant $C_{0}>0$ such that

$$
\frac{1}{M-x(t)} \leq C_{0} \quad \text { or } \quad x(t) \leq M-\frac{1}{C_{0}}, \quad \text { for all } t \in R .
$$

This implies that $\sup _{t \in R} x(t) \leq M-\frac{1}{C_{0}}<M$, which contradicts $M=\sup _{t \in R} x(t)$. Therefore, there exists $t_{0} \in R$ such that $x\left(t_{0}\right)=M=\sup _{t \in R} x(t)$.

Lemma 2.6 Supposing that $x(t) \in A P\left(R, R^{n}\right), m(x(t))=0$, then there exists $\eta_{0}>0$ such that, for every fixed $\eta_{0} \leq \eta<+\infty, \Phi(t)=\int_{0}^{t} \mathrm{e}^{-\eta(t-s)} x(s) d s$ is an almost periodic function and $\dot{\Phi}(t)$ is also an almost periodic function satisfying $\dot{\Phi}(t)=x(t)$.

Proof To $\Phi(t)$ is an almost periodic function, it suffices to show that $\Phi(t)$ is bounded. To this end, we just need show that $\int_{-\infty}^{t} \mathrm{e}^{-\eta(t-s)} x(s) d s$ is bounded.

Since $m(x(t))=0$, there exists a continuous scalar function $\epsilon(T)$ defined on $0<T<+\infty$ which satisfies $\epsilon(T) \rightarrow 0$ as $T \rightarrow+\infty$, so that $\left|\frac{1}{T} \int_{t}^{t+T} x(s) d s\right| \leq \epsilon(T)$ and, for any $T>0$, we 
have

$$
\int_{-\infty}^{t} \mathrm{e}^{-\eta(t-s)} x(s) d s=\int_{0}^{\infty} \mathrm{e}^{-\eta s} x(t-s) d s=\sum_{k=0}^{\infty} \mathrm{e}^{-\eta k T} \int_{k T}^{(k+1) T} \mathrm{e}^{-\eta(s-k T)} x(t-s) d s
$$

Let $M=\sup _{t \in R} x(t)$, then

$$
\begin{aligned}
\left|\int_{-\infty}^{t} \mathrm{e}^{-\eta(t-s)} x(s) d s\right| & \leq \sum_{k=0}^{\infty}\left[\left|\int_{k T}^{(k+1) T} x(t-s) d s\right|+M \int_{k T}^{(k+1) T}\left(1-\mathrm{e}^{-\eta(s-k T)}\right) d s\right] \\
& \leq \sum_{k=0}^{\infty}\left[T \epsilon(T)+M \int_{0}^{T}\left(1-\mathrm{e}^{-\eta s}\right) d s\right] \\
& \leq \frac{T \epsilon(T)}{1-\mathrm{e}^{-\eta T}}+M T \\
& \leq T(\vartheta(T)+M)
\end{aligned}
$$

where $\vartheta(T)=\frac{\epsilon(T)}{1-\mathrm{e}^{-\eta T T}}$ is a strictly decreasing function with respect to $T$ so that $\vartheta\left(0^{+}\right)>1$ and $\vartheta(+\infty)=0$. Thus, there is a unique $T(\eta)$ such that $\vartheta(T(\eta))=1$. Moreover, $\frac{d T(\eta)}{d \eta}<0$. In fact, $\vartheta(T(\eta))=1$ implies that

$$
\epsilon(T(\eta))-1+\mathrm{e}^{-\eta T(\eta)}=0
$$

Taking the derivative of the above inequality with respect to $\eta$ leads to

$$
\frac{d \epsilon(T(\eta))}{d T} \frac{d T(\eta)}{d \eta}-\mathrm{e}^{-\eta T(\eta)}\left[T(\eta)+\eta \frac{d T(\eta)}{d \eta}\right]=0
$$

Note that $\vartheta(T)$ is strictly decreasing, it follows that

$$
\frac{d T(\eta)}{d \eta}=\frac{\mathrm{e}^{-\eta T(\eta)} T(\eta)}{\frac{d T(\eta)}{d \eta}-\mathrm{e}^{-\eta T(\eta)}}<0
$$

Thus, $T(\eta)$ is decreasing with respect to $\eta$.

First of all, we claim that $T\left(0^{+}\right)=\infty$. If this is not true, suppose that $T\left(0^{+}\right) \leq \gamma<+\infty$. Then, for any $\eta>0$, we have

$$
1-\mathrm{e}^{-\eta T(\eta)}=\epsilon(T(\eta)) \geq \epsilon(\gamma)>0
$$

Letting $\eta \rightarrow 0^{+}$, the above inequality leads to $0>0$. This is a contradiction. Thus, $T\left(0^{+}\right)=$ $\infty$.

Second, we claim that there exists $\delta>0$ such that $T(+\infty)=\delta<+\infty$. If this is not true, suppose that $T(+\infty)=+\infty$. Then, for any $\eta>0$, we have

$$
1-\mathrm{e}^{-\eta T(\eta)}=\epsilon(T(\eta))
$$

Letting $\eta \rightarrow+\infty$, the above inequality leads to $1=1-\mathrm{e}^{-\infty}=\epsilon(+\infty)=0$. This is a contradiction. Thus, there exists $\delta>0$ such that $T(+\infty)=\delta<+\infty$. 
The fact $T(\eta)$ is decreasing with respect to $\eta$, together with $T\left(0^{+}\right)=\infty$ and $T(+\infty)=\delta<$ $+\infty$, leads to the conclusion that there exists $\eta_{0}>0$ such that, for every fixed $0<\eta_{0} \leq \eta<$ $+\infty, \delta<T(\eta) \leq T\left(\eta_{0}\right)<T\left(0^{+}\right)=+\infty$. Thus, we have

$$
|\Phi(t)| \leq\left|\int_{-\infty}^{t} \mathrm{e}^{-\eta(t-s)} x(s) d s\right| \leq T(\vartheta(T)+M)=T(\eta)(1+M) \leq T\left(\eta_{0}\right)(1+M),
$$

for $t \in R$.

The above inequality implies that, for any fixed $0<\eta_{0} \leq \eta<\infty, \Phi(t)$ is bounded. Thus, $\Phi(t)$ is an almost periodic function. Since $\dot{\Phi}(t)=x(t)$ and $x(t)$ is almost periodic, $\dot{\Phi}(t)$ is also an almost periodic function. The proof is complete.

\section{Existence of an almost periodic solution in general case}

In this section, we consider the almost periodic system

$$
\frac{d x(t)}{d t}=f(t, x(t), x(t-\tau))
$$

where $f: R \times S \rightarrow R^{n}$ is an almost periodic function in $t$ uniformly with respect to $x \in S \subset$ $R^{n}$.

In order to apply Lemma 2.4 to study the existence of the almost periodic solutions, we define the operators $L: \operatorname{Dom} L \subset X \rightarrow Z$ and $N: X \rightarrow Z$ as follows:

$$
\begin{aligned}
& X \ni x(t) \rightarrow(L x)(t)=\frac{d x(t)}{d t} \in Z, \\
& X \ni x(t) \rightarrow(N x)(t)=\left((N x)_{1}(t),(N x)_{2}(t), \ldots,(N x)_{n}(t)\right)^{T} \in Z,
\end{aligned}
$$

where

$$
(N x)_{i}(t)=f_{i}(t, x(t), x(t-\tau)), \quad i=1,2, \ldots, n .
$$

Define, respectively, the projectors $P: X \rightarrow X$ and $Q: Z \rightarrow Z$ by

$$
P x(t)=m(x(t)), \quad Q z(t)=m(z(t)), \quad x \in X, z \in Z .
$$

From the above definitions, it is obvious that the domain of $L$ in $X$ is actually the whole space, and

$$
\begin{aligned}
& \operatorname{Ker} L=\{x(t) \in X \mid L x(t)=0, \text { i.e. } \dot{x}(t)=0\}=R^{n}, \\
& \operatorname{Im} L=\{L x(t) \mid x(t) \in X\} \text { is closed in } Z, \\
& \operatorname{Ker} Q=\{z(t) \in Z \mid m(z(t))=0\} .
\end{aligned}
$$

Then we have the following results.

Lemma 3.1 Suppose that the operators $L, P, Q$ are as defined above. Then the following hold:

(i) $\operatorname{Im} P=R^{n}=\operatorname{Ker} L$; 
(ii) $\operatorname{Im} L=\operatorname{Ker} Q=\operatorname{Im}(I-Q)$;

(iii) $\operatorname{dimKer} L=\operatorname{codim} \operatorname{Im} L=n<+\infty$.

Proof By the definition of $P$ and $\operatorname{Ker} L$, (i) follows immediately. Noting that $\operatorname{dim} \operatorname{Ker} L+$ $\operatorname{dim} \operatorname{Im} L=n$, it is easy to check that (iii) holds. Now we devote ourselves to proving (ii).

First we show $\operatorname{Im} L=\operatorname{Ker} Q$. To this end, we proceed in two steps.

Step 1. $\forall \Phi(t) \in \operatorname{Im} L, x(t) \in X$ (i.e. $x \in A P\left(R, R^{n}\right)$ and $\dot{x} \in A P\left(R, R^{n}\right)$ ) such that $\Phi(t)=$ $L x(t)=\dot{x}(t)$. Then $\Phi(t)$ is almost periodic. On the other hand, it follows from Lemma 2.1 that $x(t)$ is bounded so that $x(T)-x(0)=$ const. It is easy to check that

$$
m(\Phi(t))=m(\dot{x}(t))=\lim _{T \rightarrow+\infty} \frac{1}{T} \int_{0}^{T} \dot{x}(t) d t=\lim _{T \rightarrow+\infty} \frac{1}{T}(x(T)-x(0))=0 .
$$

Thus, $\Phi(t) \in \operatorname{Ker} Q$, which implies that $\operatorname{Im} L \subset \operatorname{Ker} Q$.

Step 2. $\forall z(t) \in \operatorname{Ker} Q$, that is, $z(t) \in A P\left(R, R^{n}\right)$ and $m(z(t))=0 . z(t) \in A P\left(R, R^{n}\right)$ implies $z(t) \in C\left(R, R^{n}\right)$. Thus, there exists an original function of $z(t)$, denoted by $\Phi(t)$ such that $\dot{\Phi}(t)=z(t)$. On the other hand, it is easy to check that, for any fixed $0<\eta_{0} \leq \eta<\infty, \Phi_{0}(t)=$ $\int_{0}^{t} \mathrm{e}^{-\eta(t-s)} z(s) d s$ is also an original function of $z(t)$ such that $\dot{\Phi}_{0}(t)=z(t)$. In fact, $\Phi(t)=$ $\Phi_{0}(t)+C$, where $C$ is an arbitrary constant.

Since $m(z(t))=0$, by Lemma 2.6, $\Phi_{0}(t)=\int_{0}^{t} \mathrm{e}^{-\eta(t-s)} z(s) d s$ is an almost periodic function and $\dot{\Phi}_{0}(t)$ is also an almost periodic function satisfying $\dot{\Phi}_{0}(t)=z(t)$. Therefore, $\Phi(t)=$ $\Phi_{0}(t)+C$ is almost periodic and $\dot{\Phi}(t)=z(t)=L \Phi(t)$ is also almost periodic. This implies that $z(t)=L \Phi(t) \in \operatorname{Im} L$. Hence, $\operatorname{Ker} Q \subset \operatorname{Im} L$.

It follows from the above steps that $\operatorname{Im} L=\operatorname{Ker} Q$.

Now we show that $\operatorname{Ker} Q=\operatorname{Im}(I-Q)=\{z(t)-m(z(t)) \mid z(t) \in Z\}$. In fact,

$\forall z(t) \in \operatorname{Ker} Q$, then $m(z(t))=0$. It follows that

$$
z(t)=z(t)-m(z(t)) \in \operatorname{Im}(I-Q)
$$

which implies that $\operatorname{Ker} Q \subset \operatorname{Im}(I-Q)$.

Conversely, $\forall F(t) \in \operatorname{Im}(I-Q)$, then there exists $z(t) \in Z$ such that $F(t)=z(t)-m(z(t))$. It follows that

$$
m(F(t))=m(z(t)-m(z(t)))=m(z(t))-m(z(t))=0,
$$

which implies that $\operatorname{Im}(I-Q) \subset \operatorname{Ker} Q$. Therefore, which implies that $\operatorname{Ker} Q=\operatorname{Im}(I-Q)$.

In a word, $\operatorname{Im} L=\operatorname{Ker} Q=\operatorname{Im}(I-Q)$. That is, (ii) holds. Thus, the proof of Lemma 3.1 is complete.

It immediately follows that $L$ is a Fredholm mapping of index zero and $L \mid \operatorname{Dom} L \cap \operatorname{Ker} P$ : $(I-P) X \rightarrow \operatorname{Im} L$ is invertible. We denote the inverse of that map by $K_{P}$, it is easy to see that the map $K_{P}: \operatorname{Im} L \rightarrow \operatorname{dom} L \cap \operatorname{Ker} P$ is given by

$$
K_{P} y(t)=\int_{0}^{t} y(s) d s-m\left(\int_{0}^{t} y(s) d s\right),
$$

or

$$
\left(K_{P} y\right)_{i}(t)=\int_{0}^{t} y_{i}(s) d s-m\left(\int_{0}^{t} y_{i}(s) d s\right) .
$$


In fact, for any $y(t) \in \operatorname{Im} L$, there exists $x(t) \in X$ such that

$$
y(t)=\frac{d x(t)}{d t} .
$$

An integration of the above inequality over $[0, t]$ leads to

$$
x(t)=x(0)+\int_{0}^{t} y(s) d s .
$$

Let $K_{P} y(t)=x(t)$. In view of $x(t) \in X, K_{P} y(t) \in \operatorname{Dom} L=X$. We ensure that $K_{P} y(t) \in \operatorname{Ker} P$, $m\left(K_{P} y(t)\right)=0$. Hence, we have

$$
0=m\left(K_{P} y(t)\right)=m(x(0))+m\left(\int_{0}^{t} y(s) d s\right),
$$

which implies

$$
x(0)=-\left(\int_{0}^{t} y(s) d s\right) .
$$

Therefore,

$$
K_{P} y(t)=\int_{0}^{t} y(s) d s-m\left(\int_{0}^{t} y(s) d s\right) .
$$

Lemma 3.2 For any open bounded subset of $X$, denoted by

$$
\Omega=\left\{x(t) \in X \mid\|x(t)\|_{1}=\|x(t)\|_{0}+\|\dot{x}(t)\|_{0}<h\right\},
$$

if there exists $\widetilde{M}>0$ such that

$$
\left\|f\left(t, x_{1}, x_{2}\right)-f\left(t, y_{1}, y_{2}\right)\right\|_{0} \leq \tilde{M}\left[\left\|x_{1}-y_{1}\right\|_{0}+\left\|x_{2}-y_{2}\right\|_{0}\right], \quad x_{1}, x_{2}, y_{1}, y_{2} \in R^{n}, t \in R,
$$

then the mapping $N$ is L-compact on $\bar{\Omega}$. Here, the constant $h$ is independent of the choice of $x(t)$.

Proof It suffices to show that $Q N(\bar{\Omega})$ is bounded and $K_{p}(I-Q) N: \bar{\Omega} \rightarrow X$ is compact. We first arrive at the result that, for the constant $M=\|f(t, x(t), x(t-\tau))\|_{0}>0$,

$$
\|Q N x\|_{0}=\|m(f(t, x(t), x(t-\tau)))\|_{0} \leq m(M)=M, \quad \text { for all } x \in \bar{\Omega},
$$

which implies that $Q N(\bar{\Omega})$ is bounded in the space $\left(Z,\|\cdot\|_{0}\right)$. Second, by (3),

$$
\begin{aligned}
& \left(K_{P}(I-Q) N x\right)(t) \\
& =\int_{0}^{t}[f(s, x(s), x(s-\tau))-m(f(s, x(s), x(s-\tau)))] d s \\
& \quad-m\left(\int_{0}^{t}[f(s, x(s), x(s-\tau))-m(f(s, x(s), x(s-\tau)))] d s\right) .
\end{aligned}
$$


We shall show that $\left(K_{P}(I-Q) N x\right)(\bar{\Omega})$ is relatively compact in the space $\left(X,\|\cdot\|_{1}\right)$. To this end, we proceed in two steps.

Step 1. We claim that, for any $x(t) \in \bar{\Omega},\left(K_{P}(I-Q) N x\right)(t)$ is bounded. In fact, for any $x(t) \in \bar{\Omega}$, then $f(t, x(t)) \in N(\bar{\Omega})$ due to the definition of the operator $N$. This implies that $f(t, x(t))-m(f(t, x(t))) \in(I-Q) N(\bar{\Omega}) \subset \operatorname{Im}(I-Q) N$. From (ii) of Lemma 3.1, we know that $\operatorname{Im}(I-Q) N=\operatorname{Im} L N$. Thus, $f(t, x(t))-m(f(t, x(t))) \in \operatorname{Im} L N=\{\operatorname{Lr}(t) \mid r(t) \in N X \subset$ $Z$, i.e. $\left.r(t) \in A P\left(R, R^{n}\right)\right\}$, which implies that there exists $r(t) \in A P\left(R, R^{n}\right)$ such that

$$
\operatorname{Lr}(t)=f(t, x(t), x(t-\tau))-m(f(t, x(t), x(t-\tau))) .
$$

This, together with (4), leads to

$$
\begin{aligned}
\left(K_{P}(I-Q) N x\right)(t) & =\int_{0}^{t} \operatorname{Lr}(s) d s-m\left(\int_{0}^{t} L r(s) d s\right) \\
& =\int_{0}^{t} \dot{r}(s) d s-m\left(\int_{0}^{t} \dot{r}(s) d s\right) \\
& =r(t)-r(0)-m(r(t)-r(0)) \\
& =r(t)-m(r(t)) .
\end{aligned}
$$

Since $r(t) \in A P\left(R, R^{n}\right)$, by Lemma 2.1 and Lemma 2.3, $r(t)$ is bounded and $\|m(r(t))\|_{0}<+\infty$. Therefore, it follows from (5) that, for any $x(t) \in \bar{\Omega}$, there exists a constant $M_{0}>0$ such that $\left\|\left(K_{P}(I-Q) N x\right)(t)\right\|_{0} \leq M_{0}$.

On the other hand, we have

$$
\left(K_{P}(I-Q) N x\right)^{\prime}(t)=\dot{r}(t)=\operatorname{Lr}(t)=f(t, x(t), x(t-\tau))-m(f(t, x(t), x(t-\tau))) .
$$

In view of the almost periodicity of $f(t, x(t)),\left(K_{P}(I-Q) N x\right)^{\prime}(t)$ is bounded for any $x(t) \in \bar{\Omega}$. That is, there exists $M_{1}>0$ such that $\left(K_{P}(I-Q) N x\right)^{\prime}(t) \leq M_{0}$.

Therefore, for any $x(t) \in \bar{\Omega}$,

$$
\left\|\left(K_{P}(I-Q) N x\right)(t)\right\|_{1} \leq M_{0}+M_{1} .
$$

Step 2. By similar arguments to [41], we can prove that $\left(K_{P}(I-Q) N x\right)(\bar{\Omega})$ is equicontinuous.

Therefore, by generalizing the famous Arzela-Ascoli theorem, $\left(K_{P}(I-Q) N x\right)(\bar{\Omega})$ is relatively compact in the space $\left(X,\|\cdot\|_{1}\right)$. The proof of this lemma is complete.

Then by Lemma 3.1, Lemma 3.2, and Lemma 2.4, we have the following results on the existence of almost periodic solutions.

Theorem 3.1 Let the operators $L, N$ be defined as above. Suppose that $f(t, x)$ is almost periodic in $t$ uniformly with respect to $x \in S$ and there exists $\widetilde{M}>0$ such that

$$
\left\|f\left(t, x_{1}, x_{2}\right)-f\left(t, y_{1}, y_{2}\right)\right\|_{0} \leq \tilde{M}\left[\left\|x_{1}-y_{1}\right\|_{0}+\left\|x_{2}-y_{2}\right\|_{0}\right], \quad x_{1}, x_{2}, y_{1}, y_{2} \in R^{n}, t \in R .
$$

If there exists a constant $h$ ( $h$ is independent of the choice of $x(t)$ ) such that the open bounded set $\Omega=\left\{x(t) \in X \mid\|x(t)\|_{1}<h\right\}$ satisfies the following conditions: 
(i) for each $\lambda \in(0,1), x \in \partial \Omega \cap \operatorname{Dom} L, L x \neq \lambda N x$;

(ii) for each $x \in \partial \Omega \cap \operatorname{Ker} L, Q N x \neq 0$;

(iii) $\operatorname{deg}\{U Q N, \Omega \cap \operatorname{Ker} L, 0\} \neq 0$.

Then system (2) has at least one almost periodic solution in $\bar{\Omega} \cap \operatorname{Dom} L$.

\section{Conclusion}

In the present paper, a new method is developed to study the existence of an almost periodic solution for the ordinary or functional differential equations. The approach is based on topological degree and novel estimation techniques for the a priori bounds of unknown solutions for $L x=\lambda N x$. This new method can be seen as a supplement of the other classical methods. The approach performed in the paper could be further generalized to investigate the existence of almost periodic oscillatory cases in some other nonlinear dynamical systems. It is believed that it can be applied to the image pattern, digital image processing, data processing, signal sparse decomposition and information technology, etc.

It should be noted that there are particular differences between this paper and previous work [41].

1. This paper considered the almost periodic solution. However, the authors in [41] studied the periodic solutions. In fact, periodic solutions are a special case of almost periodic solutions. For a periodic function, it is defined by $f(t+T)=f(t)$ for some $T$. However, for the almost periodic function, it is defined in a more complicated way. For the detailed definition of an almost periodic function, one can refer to Definition 2.1.

2. Due to the big difference between the concepts of almost periodic and periodic, the method used in [41] cannot be applied to the almost periodic case directly. So we need to prove a lot of preliminary results in Section 2. From Lemma 2.1 to Lemma 2.6, all these results are very original, they have never been studied. This is the big contribution of this paper. In fact, these results are very interesting. For periodic solutions, it is not necessary to prove these results. It is obvious for the periodic case.

3. In Section 3, it seems that the method is similar to [41]. But please note that my aim of this paper is to generalize the periodic results in [41] to the almost periodic case. In fact, the detailed proof is different. Thus, it seems similar, but it is a very different problem. For example, for the periodic case, the operators $P$ and $Q$ are defined by $P x(t)=\frac{1}{T} \int_{0}^{T} x(t) d t$, however, for the almost periodic case, we have $P x(t)=m(x(t))$.

\section{Competing interests}

The author declares that there is no conflict of interests regarding the publication of this article.

\section{Acknowledgements}

The author would like to acknowledge the financial support from the National Natural Science Foundation of China under Grant (No. 61572395 and No. 11561060), Tianshui Normal University Key Construction Subject Project (Big data processing in dynamic image).

Received: 13 December 2015 Accepted: 13 March 2016 Published online: 29 March 2016

\section{References}

1. Hale, JK: Ordinary Differential Equations. Krieger, Huntington (1980)

2. Hale, JK, Verduyn Lunel, SM: Introduction to Functional Differential Equations. Springer, New York (1993)

3. Hale, JK: Periodic and almost periodic solution of functional differential equations. Arch. Ration. Mech. Anal. 15 289-309 (1964)

4. Hale, JK, Kato, J: Phase space for retarded equations with infinite delay. Funkc. Ekvacioj 1, 11-40 (1978)

5. Fink, AM: Almost Periodic Differential Equation. Springer, Berlin (1974)

6. Fink, AM, Gatica, JA: Positive almost periodic solutions of some delay integral equations. J. Differ. Equ. 83, 166-178 (1990)

7. Fink, AM: Almost periodicity of the inverse of a fundamental matrix. Proc. Am. Math. Soc. 27, 527-529 (1970) 
8. Yoshizawa, T: Stability Theory and the Existence of Periodic Solutions and Almost Periodic Solutions. Springer, New York (1975)

9. Yoshizawa, T: Stability Properties in Almost Periodic System of Functional Differential Equations. Lecture Notes in Math., vol. 799, pp. 385-409. Springer, New York (1979)

10. Hino, Y, Naito, T, Minh, NV, Shin, JS: Almost Periodic Solutions of Differential Equations in Banach Spaces. Stability and Control: Theory, Method and Applications, vol. 15. Taylor \& Francis, London (2002)

11. Seifert, G: Almost periodic solutions for single species population equations with infinite delays. In: Differential Equations and Applications in Ecology, Epidemics, and Population Problems (Claremont, Calif., 1981), pp. 203-214. Academic Press, New York (1981)

12. Seifert, G: Semi-separated conditions for almost periodic solutions. J. Differ. Equ. 11, $247-251$ (1972)

13. Seifert, G: On almost periodic solutions for undamped systems with almost periodic forcing. Proc. Am. Math. Soc. 31(1), 104-108 (1972)

14. Seifert, G: Almost periodic solutions delay differential equations with infinite delays. J. Differ. Equ. 41, 416-425 (1981)

15. Copple, WA: Dichotomies in Stability Theory. Springer, Berlin (1978)

16. Kato, J: Uniformly asymptotic stability and total stability. Tohoku Math. J. 22, 254-269 (1970)

17. Sell, GR: Periodic solution and asymptotic stability. J. Differ. Equ. 2, 147-157 (1966)

18. Sell, GR: The Floquet problem for almost periodic linear differential equations. Lect. Notes Math. 415, 239-251 (1974)

19. He, C: Almost Periodic Differential Equations. Higher Education Press, Beijing (1992) (in Chinese)

20. Favard, J: Lecons sur les fonctions presque-périodiques. Gauthier-Villars, Paris (1933)

21. Bohr, H, Neugebauer, O: Über lineare diffentil-gleichungen mit konstanten koeffizienten und fastpeniodischer rechter seite. Nachr. Ges. Wiss. Göttingen, Math.-Phys. KI., 8-12 (1926)

22. Lakshmikantham, V, Leela, S: Differential and Integral Inequalities - Theory and Applications. Academic Press, New York (1969)

23. Gopalsamy, K: Global asymptotic stability in a almost periodic Lotka-Volterra system. J. Austral. Math. Soc. Ser. B 27 346-360 (1986)

24. Gopalsamy, K: Dynamics of an almost periodic integro-differential equation. Tohoku Math. J. 37, 323-332 (1985)

25. MuRakami, S: Almost periodic solutions of a system of integro-differential equations. Tohoku Math. J. 39, 71-79 (1987)

26. Ahmad, S: On almost periodic solutions of the competing species problems. Proc. Am. Math. Soc. 102(4), 855-865 (1988)

27. Ahmad, S: On nonautonomous Volterra-Lotka competition equations. Proc. Am. Math. Soc. 177(1), 199-204 (1993)

28. Ahmad, S, Stamova, IM: Partial persistence and extinction in N-dimensional competitive systems. Nonlinear Anal. 60 , 821-836 (2005)

29. Palmer, KJ: Exponential dichotomies for almost periodic equations. Proc. Am. Math. Soc. 101(2), 293-298 (1987)

30. Yuan, R, Hong, J: The existence of almost periodic solutions for a class of differential equations with piecewise constant argument. Nonlinear Anal. TMA 8, 1439-1450 (1997)

31. Yuan, R: The existence of almost periodic solutions of retarded differential equations with piecewise constant argument. Nonlinear Anal. 7, 1013-1032 (2002)

32. Yuan, R: Almost periodic solution of a class of semilinear wave equations with boundary dissipation. Nonlinear Anal. 50, 746-761 (2002)

33. $\mathrm{Xu}, \mathrm{B}$, Yuan, R: On the positive almost periodic type solutions for some nonlinear delay integral equations. J. Math Anal. Appl. 304, 249-268 (2005)

34. Teng, Z: Nonautonomous Lotka-Volterra systems with delays. J. Differ. Equ. 179, 538-561 (2002)

35. Teng, Z: Permanence and stability of Lotka-Volterra type $n$-species competitive systems. Acta Math. Sin. 45(5), 905-918 (2002)

36. Ait Dads, E, Ezzinbi, K: Almost periodic solution for some neutral nonlinear integral equation. Nonlinear Anal. 28 1479-1489 (1997)

37. Ait Dads, E, Ezzinbi, K: Existence of positive pseudo-almost-periodic solution for some nonlinear infinite delay integral equations arising in epidemic problems. Nonlinear Anal. 41, 1-13 (2000)

38. Chen, $\mathrm{S}$, Torrejón, R: Bifurcation of almost periodic solutions for a nonlinear integral equation with delay. Nonlinear Anal. 27, 863-877 (1996)

39. Ezzinbi, K, Hachimi, MA: Existence of positive almost periodic solutions of functional equations via Hilbert's projective metric. Nonlinear Anal. 26, 1169-1176 (1996)

40. Zhao, XQ: Global attractivity in monotone and subhomogeneous almost periodic systems. J. Differ. Equ. 187, 494-509 (2003)

41. Xia, YH, Han, M: New conditions on the existence and stability of periodic solution in Lotka-Volterra's population system. SIAM J. Appl. Math. 69(6), 1580-1597 (2009)

42. Xia, YH: Global analysis of an impulsive delayed Lotka-Volterra competition system. Commun. Nonlinear Sci. Numer. Simul. 16, 1597-1616 (2011)

43. Xia, YH, Li, J, Wong, PJY: On the topological classification of dynamic equations on time scales. Nonlinear Anal., Real World Appl. 14(6), 2231-2248 (2013)

44. Romanovski, V, Xia, YH, Zhang, X: Varieties of local integrability of analytic differential systems and their applications. J. Differ. Equ. 257, 3079-3101 (2014). doi:10.1016/j.jde.2014.06.007

45. Xia, YH, Chen, X, Romanovski, V: On the linearization theorem of Fenner and Pinto. J. Math. Anal. Appl. 400(2), 439-451 (2013)

46. Zhao, NH, Xia, Y, Liu, W, Wong, PJY, Wang, RT: Existence of almost periodic solutions of a nonlinear system. J. Appl. Anal. Comput. 3, 301-306 (2013)

47. Gaines, RE, Mawhin, JL: Coincidence Degree and Nonlinear Differential Equations. Springer, Berlin (1977)

48. Alvarez, C, Lazer, AC: An application of topological degree to the periodic competing species problem. J. Austral. Math. Soc. Ser. B 28, 202-219 (1986) 